\title{
Novel use for selective inhibitors of nuclear export in $\beta$-thalassemia: block of HSP70 export from the nucleus via exportin Xpo1 improves ineffective erythropoiesis
}

\section{Susree Modepalli ${ }^{1}$ and Shilpa M. Hattangadi ${ }^{2}$}

${ }^{1}$ Georgetown University School of Medicine, Washington DC and ${ }^{2}$ National Institutes of Health, Bethesda, MD, USA

E-mail: SHILPA M. HATTANGADI - shilpam.hattangadi@nih.gov

doi:10.3324/haematol.2020.254474

$\mathrm{T}$ halassemia is the most prevalent monogenic recessive hereditary blood disorder, with around 300,000 children affected at birth worldwide. ${ }^{1}$ Imbalance between $\alpha$ - and non- $\alpha$-globin chains results in premature death of differentiating erythroid precursors (erythroblasts), because as $\alpha$-globin tetramers accumulate and precipitate, they form inclusion bodies that cause oxidative membrane damage and destruction by apoptosis.

Premature death of differentiating erythroblasts that are dividing faster because of anemia is termed ineffective erythropoiesis, as increased precursors still results in fewer terminal erythrocytes. The hypoxic environment created by anemia leads to increases in factors such as erythropoietin (Epo) or members of the transforming growth factor-beta (TGF- $\beta$ ) family and Activin receptor-II (ActR-II) trap ligands that stimulate erythropoiesis that continues to be ineffective. ${ }^{2}$ Continued apoptosis of abnormal precursors results in hemolytic anemia, aggravating other major complications of the disease like iron overload that stem from ineffective erythropoiesis. In the current issue of Haematologica, Guillem et al. ${ }^{3}$ present a novel potential therapeutic strategy for $\beta$-thalassemia, by mitigating the deleterious effect of excess $\alpha$-globin chains through increased nuclear density of chaperone HSP70 by inhibiting its nuclear export.

Multiple novel treatment modalities have been shown to ameliorate symptoms of thalassemia. These are directed either
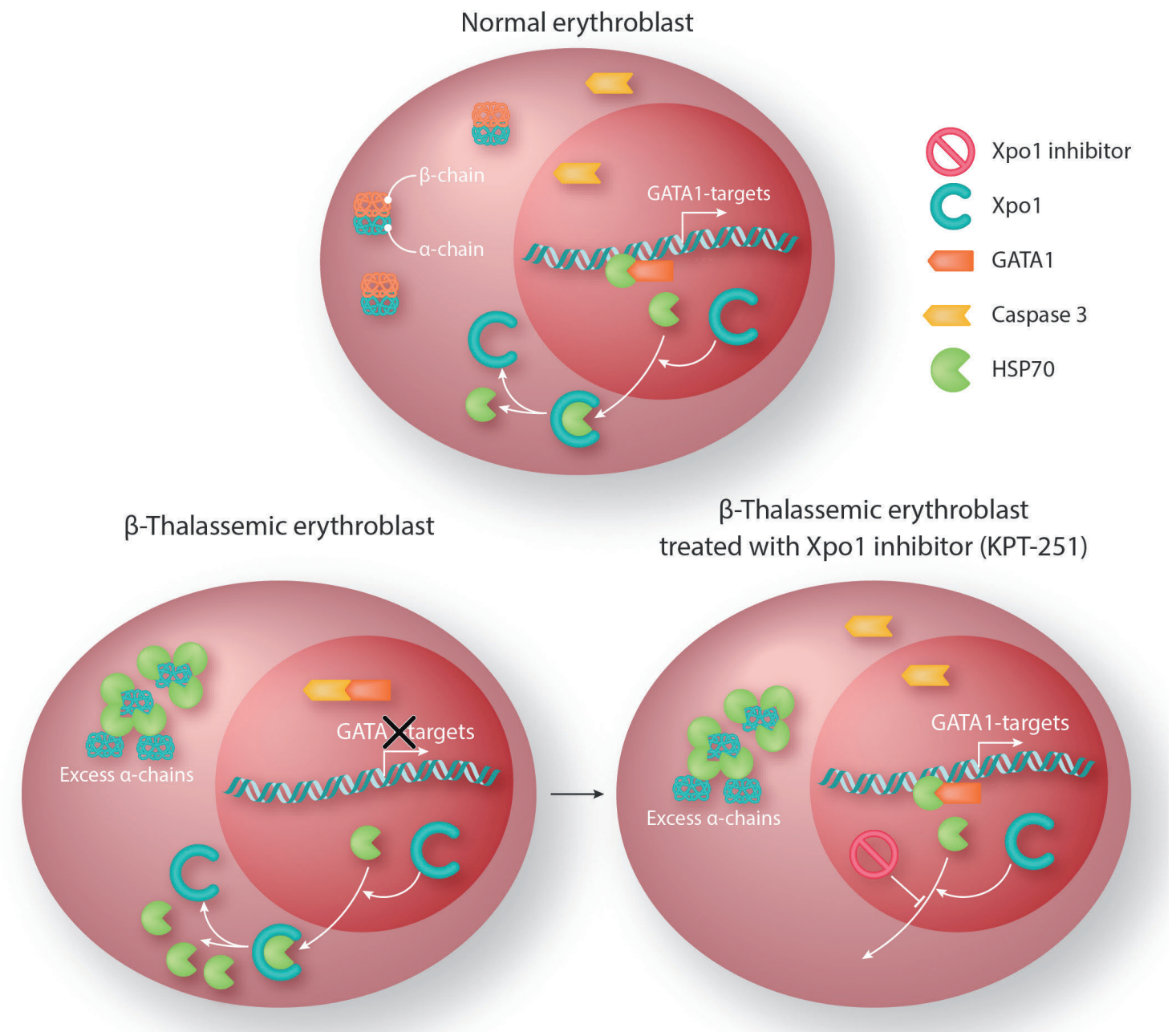

Figure 1. KPT-251 inhibition of HSP70 export from the nucleus via exportin Xpo1 improves ineffective erythropoiesis in $\beta$-thalassemia. Under normal conditions, nuclear HSP70 protects GATA1 from caspase- 3 cleavage. In thalassemia, excess free $\alpha$-globin chains sequester HSP70 in the cytoplasm, prevent normal GATA1 target expression, and result in ineffective erythropoiesis. Treating erythroblasts with the Xpo1 inhibitor KPT-251 increases nuclear levels of HSP70, rescues GATA1 from caspase-3 cleavage, and improves terminal erythroid development. 
at improving the balance between unbound $\alpha$-globin and non- $\alpha$-globin chains or correcting the ineffective erythropoiesis. Modified TFG- $\beta$ family receptor antagonists like Sotatercept (ACE-011) and Luspatercept (ACE-536) block ligand binding to ActR-II receptors, and subsequent activation of the SMAD4 signaling pathway, ${ }^{4}$ improving erythroid maturation and red cell production. Correction of the aberrant ratio of unbound $\alpha$-globin to non- $\alpha$-globin chains has been achieved by successful gene therapy by CRISPR Therapeutics, backed by Boston's Vertex Pharmaceuticals. The somatic cell therapy, named CTX001, uses edited patient's own hematopoietic stem cells (HSC) to stimulate fetal hemoglobin production. ${ }^{5}$ Targeting intracellular localization of HSP70 via XPO1 inhibition potentially merges these two treatment goals.

Several lines of evidence suggest that erythroblasts use molecular chaperones to partition unstable excess $\alpha$-globin chains during erythroid development, ${ }^{6-8}$ so it follows that targeting such chaperones could be useful in $\beta$-thalassemia when excess $\alpha$-globin tetramers accumulate and create havoc. Numerous groups have noted that the molecular chaperone Hsp70 accumulates to high levels in erythroblasts.11 and are important for streamlining erythroid maturation. ${ }^{11}$ Normal human erythroid maturation requires a transient activation of caspase- 3 at the later stages of maturation in order to prevent excessive erythrocyte production. Activated caspases can cleave GATA-1 leading to maturation arrest and/or apoptosis. ${ }^{12}$ Ribeil et al. showed that Epo causes Hsp70 to translocate into the nucleus, bind GATA-1, and protect it from caspase-3 cleavage. Conversely, during Epo deprivation, Hsp70 is excluded from the nucleus and GATA- 1 is cleaved by caspase-3, causing apoptotic death. ${ }^{13}$ Thus, alteration of the intracellular location of Hsp70 appears to play a critical role in erythroblast viability (Figure 1).

The ineffective erythropoiesis observed in $\beta$-thalassemia is characterized by accelerated erythroid differentiation, maturation arrest and apoptosis at the polychromatophilic stage. During maturation of human $\beta$-thalassemia erythroblasts, HSP70 is sequestrated in the cytoplasm (Figure 1) directly by excess free $\alpha$-globin chains. ${ }^{14}$ GATA- 1 is no longer protected, resulting in end-stage maturation arrest and apoptosis. Transduction of a nuclear-targeted HSP70 mutant or a caspase-3-uncleavable GATA-1 mutant restores terminal maturation of $\beta$-thalassemia erythroblasts. ${ }^{14}$ In this issue of Haematologica, Guillem et al. ${ }^{3}$ follow up on this mechanism to show that exportin-1 (XPO1) regulates the nucleocytoplasmic location of HSP70 in erythroid progenitors under normal conditions. Guillem et al. confirm that treating erythroblasts with the Xpo1 inhibitor KPT-251 increased nuclear levels of HSP70, rescued GATA1 from caspase-3 cleavage, and improved terminal erythroid development (Figure 1).

Although the use of selective inhibitors of nuclear export (SINE) for the treatment of lymphomas and multiple myeloma has been well reported, ${ }^{15-17}$ this is the first study showing the novel mechanism of SINE as a potential therapy for enhanced effective erythropoiesis in $\beta$ thalassemia. The clinical diversity of thalassemia makes it hard to design a one-size-fits-all therapy, and current therapies are mainly aimed at improving one or more of the underlying pathologies, most importantly transfusion dependence and iron overload. In this regard, a targeted inhibitor aimed at Xpo1 promises a more specific and low-risk treatment. Targeting HSP70 nuclear translocation in erythroid precursors represents a novel and exciting therapeutic option to ameliorate the ineffective erythropoiesis of $\beta$-thalassemia.

\section{References}

1. De Sanctis V, Kattamis C, Canatan D, et al. $\beta$-thalassemia distribution in the old world: an ancient disease seen from a historical standpoint. Mediterr J Hematol Infect Dis. 2017;9(1):e2017018.

2. Thein SL. Molecular basis of $\beta$ thalassemia and potential therapeutic targets. Blood Cells Mos Dis. 2018;70:54-65.

3. Guillem F, Dussiot M, Colin E, et al. XPO1 regulates erythroid differentiation and is a new target for the treatment of $\beta$-thalassemia. Haematologica. 2020;105(9):2240-2249

4. Makis A, Hatzimichael E, Papassotiriou I, et al. 2017 Clinical trials update in new treatments of $\beta$-thalassemia. Am J Hematol. 2016;91(11):1135-1145.

5. Lin MI, Paik E, Mishra B, et al. CRISPR/Cas9 genome editing to treat sickle cell disease and B-thalassemia: re-creating genetic variants to upregulate fetal hemoglobin appear well-tolerated, effective and durable. Blood. 2017;130(Supplement 1):284.

6. Shaeffer JR. Evidence for soluble alpha-chains as intermediates in hemoglobin synthesis in the rabbit reticulocyte. Biochem Biophys Res Comm. 1967;28(4):647-652.

7. Tavill AS, Grayzel AI, Vanderhoff GA, London IM. The control of hemoglobin synthesis. Trans Assoc Am Physicians. 1967;80:305313.

8. Olivieri NF, Weatherall DJ. Clinical aspects of beta thalassemia. In: Steinberg MH, Forget BG, Higgs DR, Nagel RL, eds. Disorders of Hemoglobin. Cambridge, United Kingdom: Cambridge University Press; 2001. pp. 277-341.

9. Morimoto R, Fodor E. Cell-specific expression of heat shock proteins in chicken reticulocytes and lymphocytes. J Cell Biol. 1984;99 (4):1316-1323.

10. Singh MK, Yu J. Accumulation of a heat shock-like protein during differentiation of human erythroid cell line K562. Nature. 1984;309(5969):631-633.

11. Banerji SS, Laing K, Morimoto RI. Erythroid lineage-specific expression and inducibility of the major heat shock protein HSP70 during avian embryogenesis. Genes Dev. 1987;1(9):946-953.

12. De Maria R, Zeuner A, Eramo A, et al. Negative regulation of erythropoiesis by caspase-mediated cleavage of GATA-1. Nature. 1999;401(6752):489-493.

13. Ribeil JA, Zermati Y, Vandekerckhove J, et al. Hsp70 regulates erythropoiesis by preventing caspase-3-mediated cleavage of GATA-1. Nature. 2007;445(7123):102-105

14. Jean-Benoît A, Ribeil JA, Guillem F, et al. HSP70 sequestration by free $\alpha$-globin promotes ineffective erythropoiesis in $\beta$-thalassaemia. Nature. 2014;514(7521):242-246.

15. Parikh K, Cang S, Sekhri A, Liu D. Selective inhibitors of nuclear export (SINE)-a novel class of anti-cancer agents. J Hematol Oncol. 2014;7:78.

16. Hing ZA, Fung HYJ, Ranganathan P, et al. Next-generation XPO1 inhibitor shows improved efficacy and in vivo tolerability in hematological malignancies. Leukemia. 2016;30(12):2364-2372.

17. Gandhi UH, Senapedis W, Baloglu E, et al. Clinical implications of targeting XPO1-mediated nuclear export in multiple myeloma. Clin Lymphoma Myeloma Leuk. 2018;18(5):335-345. 